\title{
The investigation of dye aging dynamics in writing inks using Raman spectroscopy
}

\author{
Kseniia O. Gorshkova, Ilya I. Tumkin, Liubov A. Myund, Andrey S. Tverjanovich, \\ Andrey S. Mereshchenko, Maxim S. Panov, Vladimir A. Kochemirovsky*
}

Saint Petersburg State University, 7/9 Universitetskaya nab., St. Petersburg, 199034, Russia

\section{A R T I C L E I N F O}

\section{Article history:}

Received 27 January 2016

Received in revised form

28 March 2016

Accepted 2 April 2016

Available online 7 April 2016

\section{Keywords:}

Dye

Pigment

Raman spectroscopy

Gas chromatography

Ink

\begin{abstract}
A B S T R A C T
The durability against light and aging dynamics of heteroatomic aromatic dyes and pigments containing nitrogen atoms used in writing inks was studied using Raman spectroscopy. The mechanisms of their thermal and photo-decomposition were proposed and the rates of these processes were determined. According to Raman spectroscopy, it was found that depending on the presence of one or another colorant, there are three main types (Type A, Type B and Type C) of blue dye inks used in ballpoint, gel and capillary pens which were studied in this work. Each type is characterized by a certain set of signals in Raman spectra. Time dependencies of Raman signal intensities for each type revealed the dynamics of the processes occurred with colorants included in the studied inks from the moment when they were printed on paper. Thus, the results obtained in this study can be used for the age estimation of the paper documents up to 15 years.
\end{abstract}

() 2016 Elsevier Ltd. All rights reserved.

\section{Introduction}

Despite the rapidly developing digital technologies, paper documents remain the primary source of information in many areas. A common way of document falsification is counterfeit the signatures, inscriptions and dates with ballpoint, gel and capillary pens. In some cases, in order to conceal the fraud the paper, documents are heated or irradiated by artificial and natural light sources. However, the identification of the date when a writing ink was printed on paper is one of the most difficult tasks in the field of criminology. Therefore, the study of the artificial and natural ink aging is an important criminalistic, judicial and social problem, which deserves particular attention and efforts. In order to create high-quality technique that meets all the requirements dictated by the specifics of the area under investigation, one should provide the detailed studies of all processes responsible for the property changes of ink components in time. The main components of writing inks are shown in Fig. 1. Here, the most interesting objects are dyes because they are least susceptible to temporal degradation among other components of writing inks. The proposed approach opens the possibility to identify documents up to 15 years due to

\footnotetext{
* Corresponding author.

E-mail address: lasergroupspb@gmail.com (V.A. Kochemirovsky).
}

the fact that the degradation of a dye is a long process.

When ink is applied on paper, a series of processes simultaneously occur: ink drying due to the solvent evaporation, solvent absorption on the surface of the paper and solvent diffusion inside the paper [1], ink fading due to degradation of colorants and ink solidification due to polymerization of resins [1-4]. The degradation of dyes is much slower than many other processes. This process may take several years under natural conditions due to photochemical reactions that occur during the absorption of visible or ultraviolet light. The absorption of a photon by an organic molecule leads to the formation of the electronically excited state, which results in the subsequent dye photodegradation [5-7]. Deactivation of the excited states of dyes is induced by the interaction with the environment because many radicals involved in the degradation reaction are formed from solvent or substrate upon light irradiation [8]. The photodegradation of a dye may occur due to several competing reactions:

a) N - demethylation. The methyl group of a dye is sequentially replaced by hydrogen when exposed to light [9] (Fig. 2).

b) Photooxidative cleavage of the central $\mathrm{C}$-phenyl bond occurs probably via singlet oxygen resulting in benzophenone and phenol formation $[9,10]$ (Fig. 3). It is known that the photolysis of triarylmethane dyes leads to formation of singlet oxygen not only in solution but even on paper $[8,9,11]$. As a result, in the 


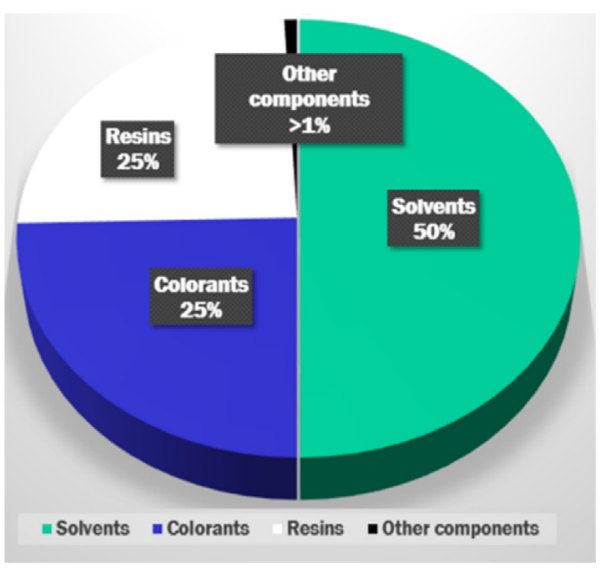

Fig. 1. The main components of writing inks [2].

absence of air under these conditions only leucobases are formed (colorless or weakly colored reduced forms of many organic dyes) [12].

c) Photoreduction of the excited dye cation to colorless leuco form. Here, the photoreduction of the excited dye cation is achieved by the addition of an electron to dye photoexcited states or by photochemical hydrogenation of a dye $[9,13]$.

It is necessary to note that dyes such as crystal violet and methyl violet are not stable and can decompose not only upon light irradiation but also in the dark due to oxidation by atmospheric oxygen. All these processes can occur at the same conditions and compete with each other. The writing inks usually consist of complex mixtures with various additives, solvents, resins. Moreover, materials of paper documents can also be very different in their structure and chemical composition. Therefore, it is quite important to study in detail the aging process of dyes taking into account all aforementioned factors [13].

\section{Experimental}

\subsection{Materials and samples}

All writing inks of various grades used in this work were commercially available and their types are presented in Supporting Information (SI, Table S1). We obtained the database of blue ink strokes of 1-cm length printed on paper at different time intervals (2000-2015 year). All samples were stored under the following conditions: temperature was kept at about $20 \pm 5{ }^{\circ} \mathrm{C}$; the relative humidity was held at 70-95\%; atmospheric pressure was $730-780 \mathrm{~mm}$ of $\mathrm{Hg}$; without direct exposure to light, UV radiation and chemicals.

In order to study the influence of environmental factors on ink aging two groups of samples were prepared. In the first group, the ink strokes with a length of about $1 \mathrm{~cm}$ were drawn on paper. In the second one, the strokes of solution of colorant in dimethyl formamide (DMFA) were drawn on paper by a glass rod. The choice of DMFA was based on its high dissolving capacity and suitability for spectroscopic studies in the near infrared region. Then all of these samples were subjected to heat and light exposure.

We used the following colorants in powder form: fat-soluble purple $\mathrm{K}$, alcohol-soluble phthalocyanine blue, direct purple, basic purple $\mathrm{K}$, acid purple, acid bright blue, pigment phthalocyanine blue, victoria blue, crystal violet, methyl violet, rhodamine G, basic blue lacquer $\mathrm{K}$.

For spectrophotometric measurements the piece of a paper the sizes of $3 \times 10 \mathrm{~mm}$ was stroked by the aforementioned inks. Also approximately the same paper area was stroked by the DMFA solutions of colorants using a glass rod.

For thin-layerchromatography (TLC), plates of Silufol (PTSH-AF$15 \times 15)$ on the aluminum substrate were used. The chromatographic separation was carried out in the next solvent system: ethyl acetate-isopropyl alcohol-water-acetic acid in a volume ratio of 30:15:10:1. The about $1 \mathrm{mg}$ of each individual colorant was placed in microvial and dissolved in the $2 \mathrm{ml}$ of DMFA for $2 \mathrm{~h}$ at the room temperature. The resulting dye solutions were transferred to the starting line on chromatographic plate using a microcapillary pipet. Before the chromatographic measurements, the TLC was stored at room temperature for $24 \mathrm{~h}$. The zone detection was carried out visually in visible light.

\subsection{Instrumentation}

Raman spectra were obtained using a Senterra Raman spectrometer (Bruker Optics) equipped with three lasers with excitation wavelengths of 488,532, $785 \mathrm{~nm}$, a laser module confocal

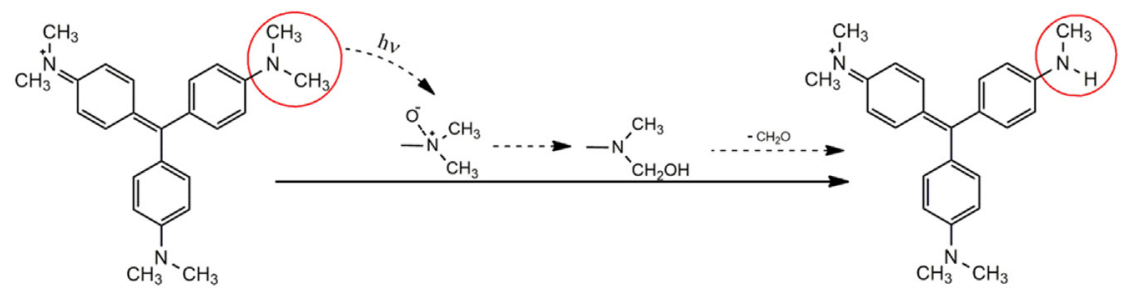

Fig. 2. The mechanism of N-demethylation.

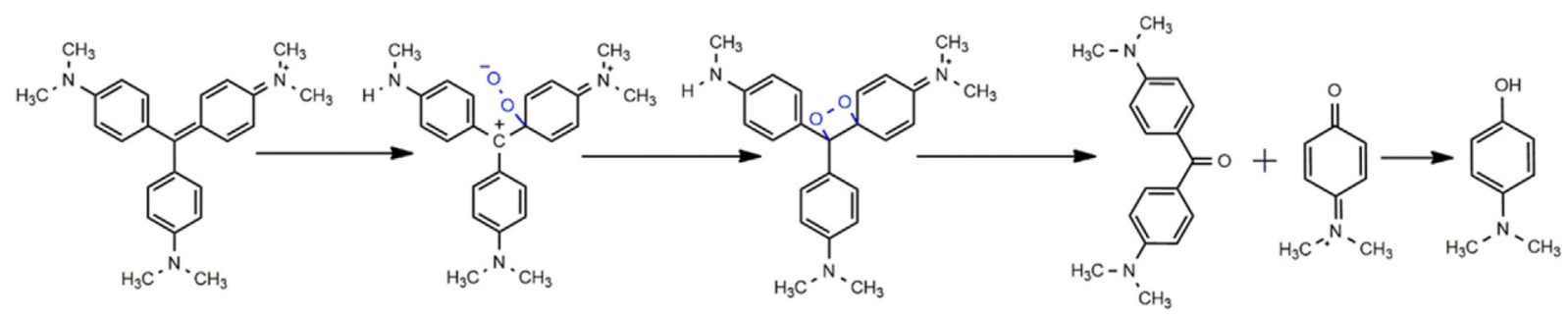

Fig. 3. The mechanism of the degradation of methyl violet through the attack of singlet oxygen. 
microscope (Olympus) and the fiber-optic sensor for spectra recording in an external sample compartment. A fully confocal system is able to operate at three different exciting wavelengths providing the highest possible spatial resolution at the same time. Raman spectra were recorded in the range of $440-1735 \mathrm{~cm}^{-1}$. The baseline correction (two iterations) and smoothing (twenty five iterations) were performed for processing Raman spectra.

The electronic absorption spectra were recorded on a dual-beam scanning spectrophotometer Lambda 1050 with double monochromator using the module-integrating sphere of $150 \mathrm{~mm}$ (InGaAs). The measurements were carried out in the wavelength range of $450-850 \mathrm{~nm}$. The spectral resolution was $2 \mathrm{~nm}$.

Gas chromatographic analysis was performed using a Chromatec-Crystal 5000.2 chromatograph with dispenser for solid samples and a flame ionization detector. Analysis was performed using instrument parameters given in SI (Table S2). The strips of paper with studied ink strokes (each $1 \mathrm{~cm}$ long) were placed into the evaporator of the gas chromatograph using the dispenser for solid samples and kept there for $1 \mathrm{~min}$.

To study thermal effects all samples were kept in the thermostat at $60^{\circ}$ for the $7.25 ; 22.75 ; 35.25 ; 43.75 \mathrm{~h}$ and at $90^{\circ}$ for $8.5 ; 23.5$; $32.5 ; 56 \mathrm{~h}$. To study the light effect all samples were irradiated with a xenon arc lamp (Oriel Instruments 67005 (output power $3.5 \mathrm{~W}$ )) for different times: 1, 3, 10, 30, 100 and 300 min. Alternatively, the studied samples were irradiated by the 355, 532 and 661-nm lasers. For normalization of obtained results we introduced the parameter $E$ which was calculated using the next equation:

$E=\frac{N}{S} \times t$

$N$ - the output power measured using a power meter, W.; $S$ - the area of the spot formed by the focused irradiation, $\mathrm{m}^{2} ; t-$ the irradiation time, $s$.

\section{Results and discussion}

Raman spectroscopy requires a selection of parameters in order to obtain spectra for samples of different kinds. In particular, one should take into account a number of criteria when working with inks deposited on paper. Thus, such criteria as laser power, exposure time and number of scans were optimized to ensure the best ratio signal-to-noise for all studied samples. Moreover, the absence of laser-induced degradation of the samples was also taken into consideration as another very important criterion. The lens of 50 times magnification used in the Raman experiments allowed to analyze the fully shaded portion of the ink stroke. As a result, a stroke size was sufficient enough for taking into account the inhomogeneity of ink distribution caused by the specificity of paper. In order to understand the influence of paper, the Raman spectra of paper substrates at each wavelength were recorded separately and then were subtracted from the Raman spectra of the ink containing samples.

\subsection{Classification of writing inks}

The Raman spectra of writing inks showed that colorants (dyes and pigments) have the most pronounced peaks, which were used for the classification of the samples. Thus, all the studied samples can be divided into three types of the Raman spectra recorded at $785 \mathrm{~nm}$ (Fig. 4) based on the presence of a characteristic group of peaks that correspond to one or another colorant (SI, Figs. S1-S3, Tables S3-S5).

However, writing inks attributed to the type B do not give distinct spectral characteristics at the wavelength of $785 \mathrm{~nm}$ due to

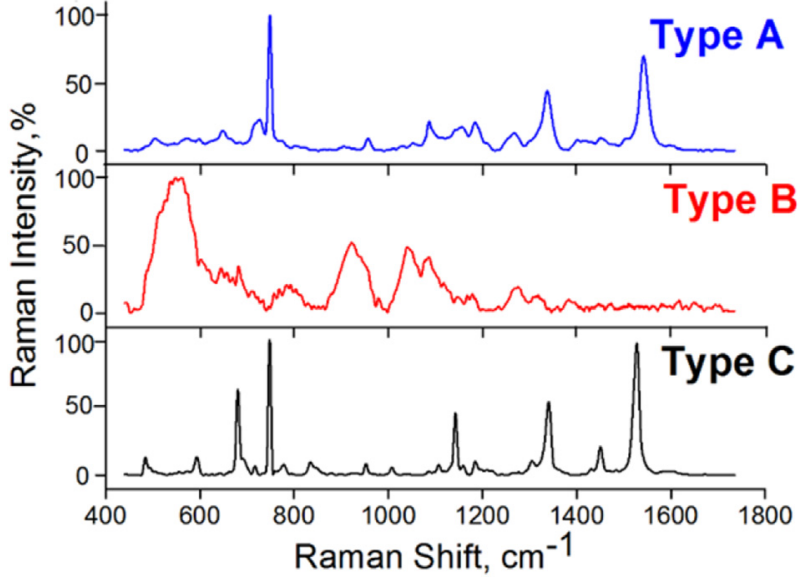

Fig. 4. A series of Raman spectra of blue writing ink of different brands printed on paper are classified as types A, B and C. All spectra were recorded using $785 \mathrm{~nm}$ laser.

strong contribution of luminescence. As a result, the useful Raman signal is too small to detect. Therefore, in order to conduct further analysis of samples of this type the Raman spectra were recorded at $532 \mathrm{~nm}$ (SI, Fig. S2, Table S4). According to the interpretation of the obtained Raman spectra along with the results of TLC and spectrophotometric experiments, the following types of colorants were proposed:

1 Type A - the alcohol-soluble phthalocyanine blue mixed with the triarylmethane group of dyes (such as basic violet, crystal violet, methyl violet, fat-soluble purple $\mathrm{K}$, acid bright blue, victoria blue).

2 Type $\mathrm{B}-$ the triarylmethane group of dyes.

3 Type $\mathrm{C}$ - the phthalocyanine blue pigment.

The spectrophotometric analysis of the samples (Table 1) confirmed the assumption that the Raman spectra obtained at different wavelengths are significantly different due to the influence of resonance (or preresonance) effects.

When the excitation frequency is close to the frequency of electronic-vibration transition an increase of scattering light occurs. This is especially important for the investigation of the multicomponent mixtures which have Raman spectra with hundreds of bands. When the wavelengths corresponding to the absorption maximum or near maximum in electronic spectra of colorants are applied, the optically active modes associated with chromophore group are substantially enhanced. In this regard, there is a possibility of selective isolation and identification of the substance in the studied matrix.

The Raman spectra recorded at $785 \mathrm{~nm}$ excitation show that the most intense bands correspond to the vibrations of the macrocyclic conjugated $\pi$-system which contains nitrogen atoms and carbon atoms of the central benzene ring in molecule of copper phthalocyanine. On the other hand, the most prominent bands of the Raman spectra obtained at $532 \mathrm{~nm}$ excitation correspond to the symmetric vibrations of the chromogen (Fuchsonimin) in triarylmethane dyes (Table 1) [14-22]. Furthermore, as it was proposed, the A-type writing inks contain a mixture of dyes and, therefore, if one apply the excitation wavelength near the absorption maximum of one or another dye it is possible to distinguish the peaks corresponding to each individual dye separately.

\subsection{Natural aging}

The temporal dependence of the ratios of the characteristic peak 
Table 1

The absorption maxima of the components of writing inks printed on paper.

\begin{tabular}{lll}
\hline Type & Colorant & $\lambda_{\max }(\mathrm{nm})$ \\
\hline A & alcohol-soluble phthalocyanine & $610-620$ \\
& & $670-680$ \\
& triarylmethane group of dyes & $530-580$ \\
B & triarylmethane group of dyes & $530-580$ \\
& - & $\sim 650$ \\
C & phthalocyanine blue pigment & $670-680$ \\
& - & $\sim 550$
\end{tabular}

intensities for each type of writing ink is presented in Fig. 5.

Here, it should be pointed out that the peak intensities in the Raman spectra are also dependent on the concentration of the studied inks in the focal spot, which is impossible to control in our experiments. Therefore, we used not the absolute values of the peaks but the ratio of their intensities in order to eliminate the concentration influence [23]. For this purpose, the pairs of characteristic peaks were chosen as follows (Table 2): the first peak corresponds to the peripheral vibrations of bonds which are most susceptible to change during time and the second peak corresponds to the vibrations of the most stable bonds (usually stretching vibrations involving the carbon in the chromophore group).

In Fig. 5, each point on the graphs represents the average value of the ratios of the corresponding peaks obtained from five independent measurements of single sample. Herein, the X-axis is the time in months starting from the date when the writing ink was applied on a paper and up to the moment when the spectrum was taken. We believe that the complex form of the dependence is due to the change of dominant processes in a certain interval.

\subsection{Artificial aging}

\subsubsection{Aging induced by the irradiation with a xenon lamp}

The xenon lamp was used to simulate ink aging process because its emission spectrum is close to the sunlight spectrum and, therefore, used to mimics daylight. The writing inks and pure colorants were deposited on paper $1 \mathrm{~h}$ before the irradiation ("fresh" ink strokes). Fig. 6 illustrates the dependencies of the ratios of the characteristic peaks on the irradiation time. These dependencies are demonstrated to be similar to those obtained in the case of the natural aging of the samples (Fig. 5). The presence of the first maximum in Fig. 6B could be caused by the influence of colorant matrix because solvents.

Resins can also contribute to the peak intensities in the Raman spectra. On the other hand, this maximum was not observed for the pure colorant (Fig. 6A). Furthermore, Fig. 6A and B demonstrate similar behavior of these dependencies after $10 \mathrm{~min}$ of irradiation, probably meaning that the most likely further course of the curves is determined by only the degradation of colorants. Table 3 presents the correlation between the time of irradiation with a xenon lamp and the natural aging time obtained by comparison of the ratios of the peak intensities taken from Fig. 6 at the corresponding irradiation time with those shown in Fig. 5.

Triarylmethane dyes corresponding to B-type are less resistant to irradiation with respect to phthalocyanine dyes. Thus, Raman spectra exhibit no clearly distinguishable peaks after $30 \mathrm{~min}$ of irradiation; as a result, it is impossible to calculate the analytical ratios of the peak intensities.

The spectral range corresponding to the fastest dye degradation was determined. First, it was shown that the dependencies obtained after irradiation of the samples by the unfocused laser beam (Fig. 7) have the same shape as the initial part of those observed after irradiation with a xenon lamp (Fig. 6). The points on the curves corresponding to the ink aging experiments with a xenon lamp are a superposition of the ratios obtained after irradiation with lasers at certain wavelengths (Fig. 7).

Second, the shape of the dependencies for the samples acquired after laser irradiation at a wavelength of $355 \mathrm{~nm}$ has a more extreme character (more pronounced maxima) than those observed at wavelength of 532 and $661 \mathrm{~nm}$ despite the fact that the latter are near the absorption maxima of colorants included in the writing ink composition. Therefore, it can be assumed that the dye degradation is mainly caused by the absorption of light in the UV range.

\subsubsection{Aging caused by the temperature effect}

The aging of dyes may be induced not only by the influence of light (photo-degradation) but also by temperature (thermodegradation). In this regard, in order to clarify the role of temperature in the ink aging process, the "fresh" ink strokes deposited on paper no later than $1 \mathrm{~h}$ prior the experiment were kept at different temperatures for a certain time. The overall results of this experiment are shown in Fig. 8.

The dependencies of the characteristic peaks on the heating time are linear with a small slope angle (Fig. 8A) for pure colorants, which may indicate a minor influence of temperature on the colorant degradation. Fig. 8B demonstrates that the curves characterizing the temperature effect for writing inks also exhibit linear behavior and the monotonic growth similarly to the initial part of the curves obtained in the natural aging experiments (Fig. 5). Thus, the aforementioned experiment indicates that the dye matrix is responsible for the increase of value of the peak ratios.
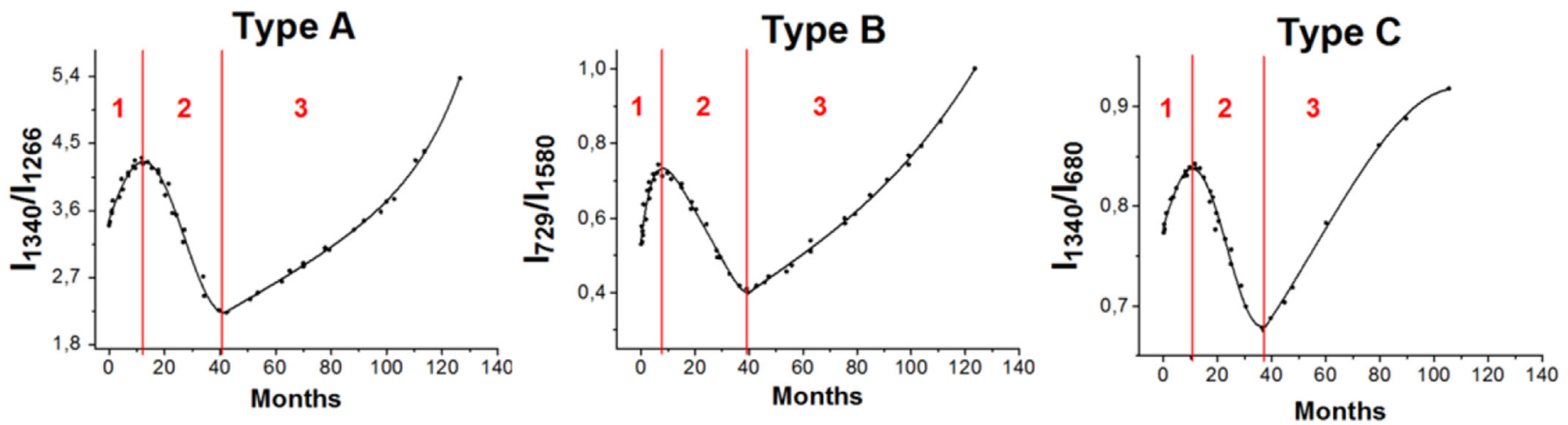

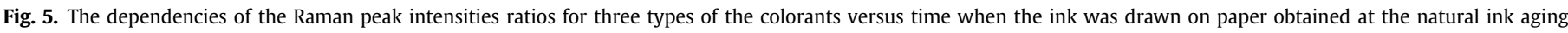
conditions. 
Table 2

The characteristic Raman peaks chosen to obtain the temporal dependencies.

\begin{tabular}{|c|c|c|c|c|}
\hline Type & Colorant & Raman peak $\left(\mathrm{cm}^{-1}\right)$ & Vibration $(v)$ & Peak ratio \\
\hline \multirow[t]{2}{*}{ A } & alcohol-soluble phthalocyanine & 1340 & $\begin{array}{l}(\mathrm{C}-\mathrm{C})_{\text {ring }} \\
(\mathrm{C}-\mathrm{N})\end{array}$ & $1340 / 1266$ \\
\hline & & 1266 & $\mathrm{SO}_{2}$ & \\
\hline \multirow[t]{2}{*}{ B } & triarylmethane group of dyes & 729 & $(\mathrm{C}-\mathrm{N})$ & $729 / 1587$ \\
\hline & & 1587 & $(\mathrm{C}-\mathrm{C})_{\text {ring }}$ & \\
\hline $\mathrm{C}$ & phthalocyanine blue pigment & 1340 & $\begin{array}{l}(\mathrm{C}-\mathrm{C}) \text { ring } \\
(\mathrm{C}-\mathrm{N})\end{array}$ & $1340 / 680$ \\
\hline & & 680 & $(\mathrm{C}-\mathrm{C}-\mathrm{H})$ & \\
\hline
\end{tabular}
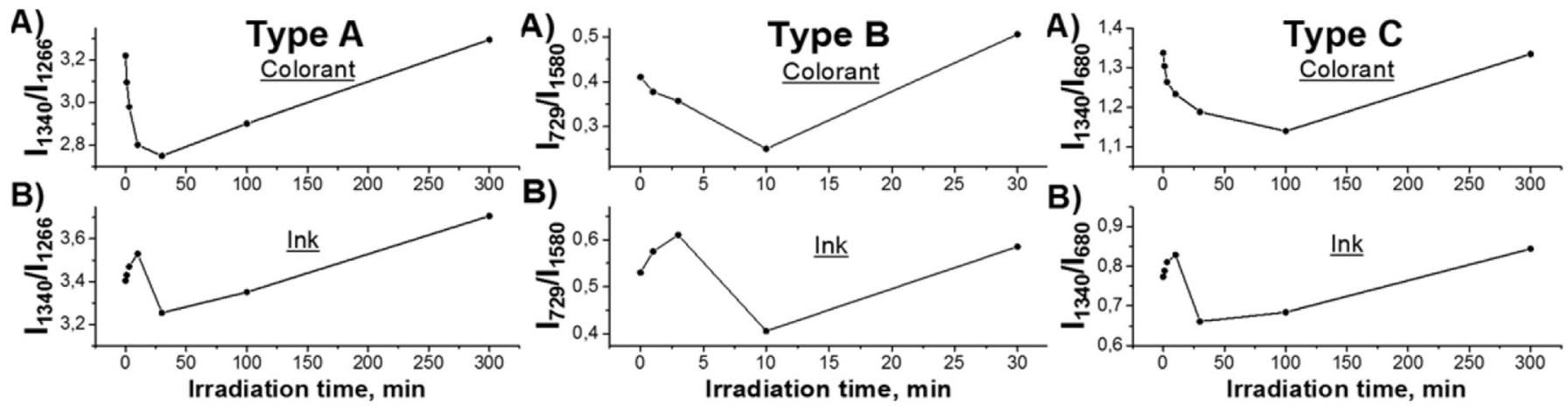

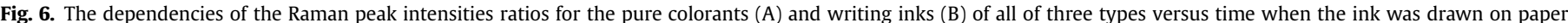
obtained at the artificial ink aging conditions (irradiation with a xenon lamp).

Table 3

The correlation between the time of irradiation with a xenon lamp and natural aging time.

\begin{tabular}{lccc}
\hline Irradiation time, min & \multicolumn{3}{c}{ The natural aging time, months } \\
\cline { 2 - 4 } & $\mathrm{A}$ & $\mathrm{B}$ & $\mathrm{C}$ \\
\hline 0 & 0.00 & 0.00 & 0.00 \\
1 & 0.23 & 0.47 & 0.95 \\
3 & 0.47 & 1.00 & 3.70 \\
10 & 1.00 & 39.00 & 5.20 \\
30 & 27.00 & 73.00 & 36.53 \\
100 & 91.00 & - & 38.82 \\
300 & 102.00 & - & 73.87 \\
\hline
\end{tabular}

tetraethylene glycol, glycerol and 2-phenoxyethanol during the storage at the natural conditions.

It is shown here that the solvent evaporation occurs at a substantial speed only in the first month after ink deposition (about $90 \%$ of solvent evaporates) then the evaporation process slows down dramatically. The presence of solvents in the ink stroke becomes insignificant after six months of the natural aging. This time interval matches the initial part of the curves observed in Fig. 5. Analogically, the temperature influence on the artificial ink aging presented as a monotonic growth of the curve in Fig. 8B can also be explained by the solvent evaporation process. It is important observation because,

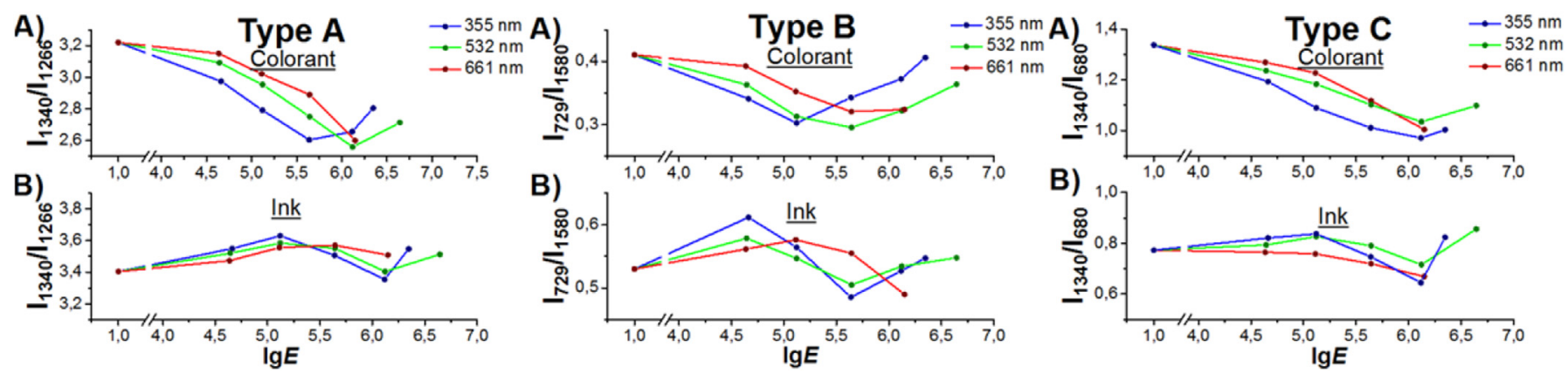

Fig. 7. The dependencies of the Raman peak intensities ratios for the pure colorants (A) and writing inks (B) of all of three types versus the logarithm of the parameter $E$.

\subsection{Gas chromatography measurements}

Gas chromatography was used to establish the time interval of the thermal degradation processes of the writing inks stored at natural conditions, in particular the solvent evaporation and decomposition of the ink components. Fig. 9 demonstrates the change of the chromatographic peak area corresponding to for example, the presence of tetraethylene glycol determines the peak intensity at $1266 \mathrm{~cm}^{-1}$, which in turn was used to plot corresponding dependencies in Fig. 8B. In this case, it is also necessary to take into account the presence of other solvents in the composition of inks. In addition, the growth of the initial part of the curve observed for the samples irradiated with a xenon lamp (Fig. 6B) and the unfocused laser beam (Fig. 7B) can be explained similarly. 


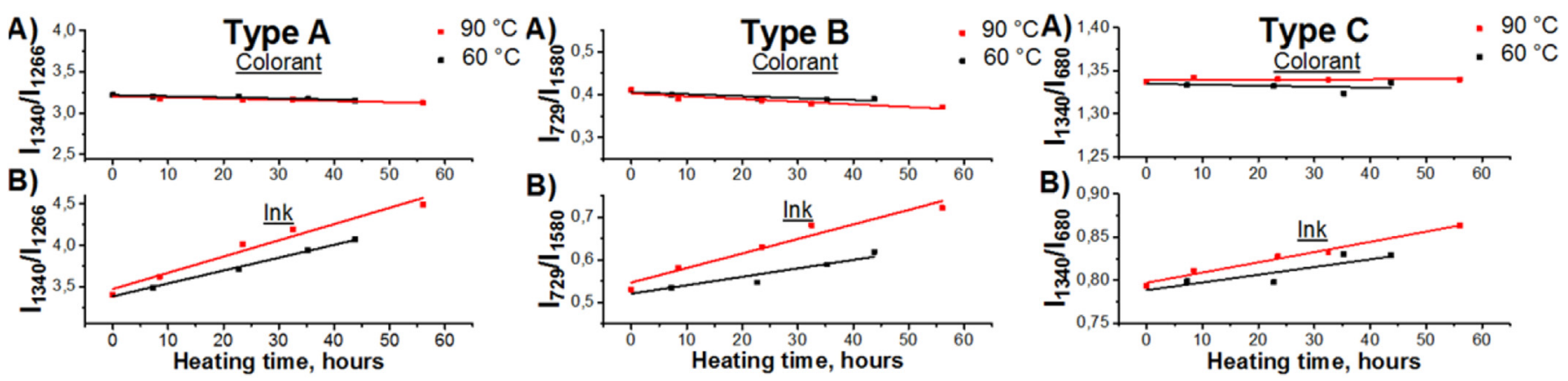

Fig. 8. The dependencies of the Raman peak intensities ratios for the pure colorants (A) and writing inks (B) of all of three types versus the heating time.

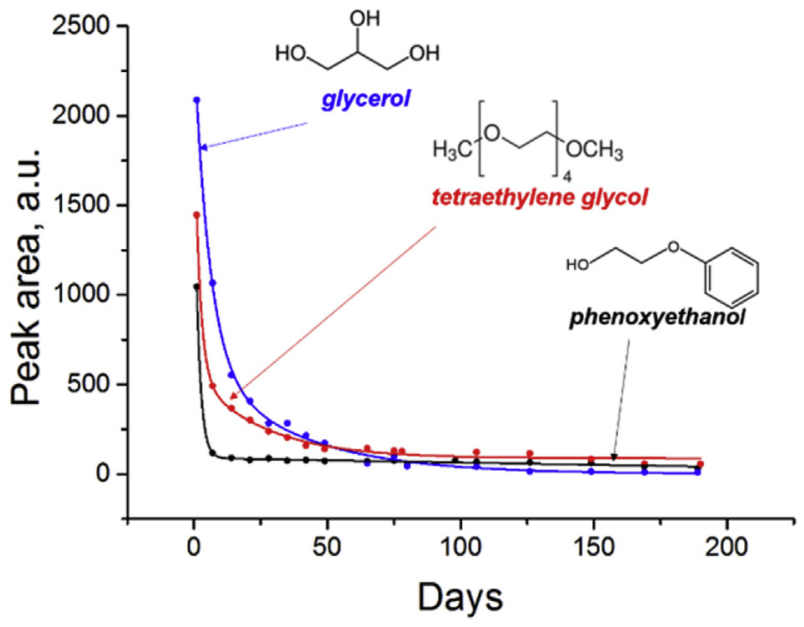

Fig. 9. The change of the chromatographic peak area corresponding to tetraethylene glycol, glycerol and 2-phenoxyethanol with the storage time at the natural conditions.

\subsection{Dye aging model}

Based on overall results discussed above, the dye degradation model is proposed. As an example let us consider the B-type colorant, which is based on the triarylmethane group of dyes. In order to plot the peak intensity ratios versus the exposition time (Fig. 10), Raman peaks at $729 \mathrm{~cm}^{-1}$ corresponding to $\mathrm{C}-\mathrm{N}$ vibrations and $1580 \mathrm{~cm}^{-1}$ corresponding to the complex $\mathrm{C}-\mathrm{C}$ variations of the chromogen (Fuchsonimin) were used.

These vibrations are sensitive to degradation in different extent due to specific nature of the Raman band. The graph shown in Fig. 10 can be split in three parts responsible for one or another dominant process:

The first part $\left(\Delta t_{1}\right)$ - the transformation of the dye matrix (volatile components evaporation, resin solidification).

The second part $\left(\Delta t_{2}\right)-\mathrm{N}$ - demethylation (the methyl group of the dye is consistently replaced by hydrogen upon light irradiation).

The third part $\left(\Delta t_{3}\right)$ - the photooxidative cleavage of the central $\mathrm{C}-$ phenyl bond resulting in formation of benzophenone and phenol.

Fig. 10 illustrates the combination of the aforementioned processes dominating in certain time intervals.

\section{Conclusion}

The investigation of natural and artificial aging of writing inks

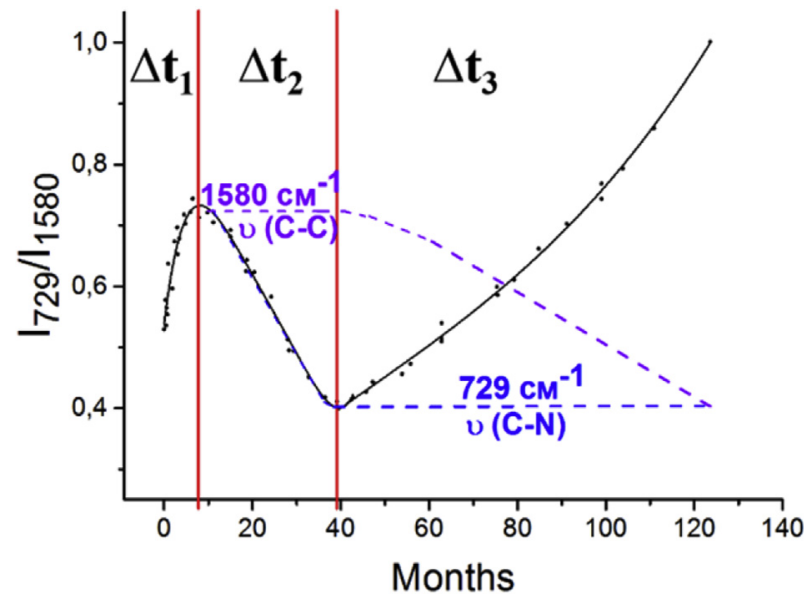

Fig. 10. The dependencies of the Raman peak intensities ratios for the B-type colorant versus time when the ink was drawn on paper.

printed on paper using Raman spectroscopy was performed. It was found out that the colorants determines the Raman spectra dynamics of the writing inks except for the initial period of ink aging process, which is accompanied by evaporation of volatile ink components. In the natural and artificial aging experiments, the key factor of long term degradation of the colorants and inks composed of them is light exposure. The impact of other components on the Raman spectral characteristics of the studied inks is manifested only within the initial period after ink deposition on paper (up to 6 months). The temperature within the range of $90-95{ }^{\circ} \mathrm{C}$ has no significant effect on the Raman spectral properties of the colorants and corresponding dyes. Based on the obtained dependencies of the Raman peak intensities ratios versus the time exposure, the dye degradation model was proposed. It was suggested that there are several competing bond-breaking and bond-making reactions corresponding to the characteristic vibration frequencies of the dye molecule that simultaneously occur during ink aging process. Thus, the overall results of this work showed that Raman spectroscopy is an effective tool for studying the aging processes of the writing inks drawn on paper more than half a year after treatment. Moreover, these results can be implemented to develop the novel and promising method of criminology.

\section{Acknowledgments}

Authors acknowledge Saint-Petersburg State University for the financial support. We would like to thank E.R. Rossinskaya and Yu.G. Korukhov from Chamber of Judicial Experts ("Sudex", Moscow) for their expertise and contribution to this project. The authors also express their gratitude to Centre for Optical and Laser Materials 
Research and Chemistry Educational Centre of Saint Petersburg State University.

\section{Appendix A. Supplementary data}

Supplementary data related to this article can be found at http:// dx.doi.org/10.1016/j.dyepig.2016.04.009.

\section{References}

[1] Weyermann C, Almog J, Bügler J, Cantu AA. Minimum requirements for application of ink dating methods based on solvent analysis in casework Forensic Sci Int 2011;210:52-62. http://dx.doi.org/10.1016/ j.forsciint.2011.01.034.

[2] Weyermann C, Schiffer B, Margot P. A logical framework to ballpoint ink dating interpretation. Sci Justice 2008;48:118-25. http://dx.doi.org/10.1016/ j.scijus.2007.10.009.

[3] Ezcurra M, Góngora JMG, Maguregui I, Alonso R. Analytical methods for dating modern writing instrument inks on paper. Forensic Sci Int 2010;197:1-20. http://dx.doi.org/10.1016/j.forsciint.2009.11.013.

[4] Lociciro S, Dujourdy L, Mazzella W, Margot P, Lock E. Dynamic of the ageing of ballpoint pen inks: quantification of phenoxyethanol by GC-MS. Sci Justice 2004;44:165-71. http://dx.doi.org/10.1016/S1355-0306(04)71709-8.

[5] Stepanov BI. Introduction to chemistry and technology of organic dyes. Moscow: Chemistry; 1984.

[6] Coyle JD. Introduction to organic photochemistry. England: John Wiley \& Sons; 1986.

[7] Dickert F, Becker HGO. 3., bearbeitete Auflage. In: Bötcher H, Dietz F Rehorek D, Roewer G, Schiller K, Timpe H-J, editors. Einführung in die Photochemie. Berlin: Deutscher Verlag der Wissenschaften; 1991. http:// dx.doi.org/10.1002/bbpc.19930970423. ISBN 3-326-00604-7, 503 Seiten, Preis: DM 120,-. Berichte Der Bunsengesellschaft Für Phys Chemie 1993;97: 643-643.

[8] Brezová V, Pigošová J, Havlınová B, Dvoranová D, Durovič M. EPR study of photochemical transformations of triarylmethane dyes. Dye Pigment 2004;61: 177-98. http://dx.doi.org/10.1016/j.dyepig.2003.10.012.

[9] Caine MA, McCabe RW, Wang L, Brown RG, Hepworth JD. The influence of singlet oxygen in the fading of carbonless copy paper primary dyes on clays. Dye Pigment 2001;49:135-43. http://dx.doi.org/10.1016/S0143-7208(01) 00021-3.

[10] Kuramoto N, Kitao T. The contribution of singlet oxygen to the photofading of triphenylmethane and related dyes. Dye Pigment 1982;3:49-58. http:// dx.doi.org/10.1016/0143-7208(82)80012-0.

[11] Caine MA, McCabe RW, Wang L, Brown RG, Hepworth JD. The inhibition of triphenylmethane primary dye fading in carbonless copying paper systems by singlet oxygen quenching bis(dithiocarbamato)nickel(II) complexes. Dye Pigment 2002;52:55-65, http://dx.doi.org/10.1016/S0143-7208(01)00076-6.

[12] Borodkin WF. Chemistry of dyes. Moscow: Chemistry; 1981.

[13] Weyermann C, Kirsch D, Vera CC, Spengler B. Evaluation of the photodegradation of crystal violet upon light exposure by mass spectrometric and spectroscopic methods. J Forensic Sci 2009;54:339-45. http://dx.doi.org/ 10.1111/j.1556-4029.2008.00975.x.

[14] Ziminov AV, Ramsh SM, Spiridonov IG, Yourre TA, Butkhuzi TG, Turiev AM. Synthesis and research of physical and chemical properties of phthalocyanines complexes with dand f-elements. Vestn Saint-Petersbg Univ; 2009. p. 95-109.

[15] Rosa A, Baerends EJ. Metal-macrocycle interaction in phthalocyanines: density functional calculations of ground and excited states. Inorg Chem 1994;33: 584-95. http://dx.doi.org/10.1021/ic00081a029.

[16] Mack J. Assignment of the optical spectra of metal phthalocyanines through spectral band deconvolution analysis and ZINDO calculations. Coord Chem Rev 2001;219-221:993-1032. http://dx.doi.org/10.1016/S0010-8545(01) 00394-0.

[17] Panda DH. A concise guide on textile dyes, pigments and dye intermediates with textile printing technology. Niir Project Consultancy Services; 2013.

[18] Reichenbächer M. Challenges in molecular structure determination. 2012. http://dx.doi.org/10.1007/978-3-642-24390-5.

[19] García Río L, Godoy A. Influence of CTACl cationic micelles on the spectral behavior of crystal violet. Chem Phys 2006;327:361-7. http://dx.doi.org/ 10.1016/j.chemphys.2006.05.008.

[20] Maruyama Y, Ishikawa M, Satozono H. Femtosecond isomerization of crystal violet in alcohols. J Am Chem Soc 1996;118:6257-63. http://dx.doi.org/ $10.1021 /$ ja960024z.

[21] Oliveira CS, Branco KP, Baptista MS, Indig GL. Solvent and concentration effects on the visible spectra of tri-para-dialkylamino-substituted triarylmethane dyes in liquid solutions. Spectrochim Acta Part A Mol Biomol Spectrosc 2002;58:2971-82. http://dx.doi.org/10.1016/S1386-1425(02) 00044-6.

[22] Cañamares MV, Chenal C, Birke RL, Lombardi JR. DFT, SERS, and singlemolecule SERS of crystal violet. J Phys Chem C 2008;112:20295-300. http:// dx.doi.org/10.1021/jp807807j.

[23] Petrov AA, Pushkarev EA. Correlation spectral analysis of substances. Saint Petersburg: Chemistry; 1993. 